Portland State University

PDXScholar

$9-1-1987$

\title{
Energy-transfer theory for the classical decay rates of molecules at rough metallic surfaces
}

P.T. Leung

Portland State University

Thomas F. George

Follow this and additional works at: https://pdxscholar.library.pdx.edu/phy_fac

Part of the Physics Commons

Let us know how access to this document benefits you.

\section{Citation Details}

Leung, P. T., \& George, T. F. (1987). Energy-transfer theory for the classical decay rates of molecules at rough metallic surfaces. Physical Review B (Condensed Matter), 36(9), 4664-4671.

This Article is brought to you for free and open access. It has been accepted for inclusion in Physics Faculty Publications and Presentations by an authorized administrator of PDXScholar. Please contact us if we can make this document more accessible: pdxscholar@pdx.edu. 


\title{
Energy-transfer theory for the classical decay rates of molecules at rough metallic surfaces
}

\author{
P. T. Leung and Thomas F. George \\ Department of Physics and Astronomy and Chemistry, 239 Fronczak Hall, \\ State University of New York at Buffalo, Buffalo, New York 14260
}

(Received 24 April 1987)

\begin{abstract}
The problem of the decay rates for molecules at rough metallic surfaces is considered, where the classical electromagnetic energy-transfer theory of Chance, Prock, and Silbey for a flat surface is generalized to the case of a rough boundary. A dynamical theory is constructed through the combination of the Sommerfeld antenna theory and the integral equation formalism of Maxwell's equations at rough boundaries established mainly by Maradudin, Mills, and Agarwal. Perturbative solutions are obtained and numerical results are given with reference to a shallow sinusoidal grating surface. The results, when compared with those obtained previously from the application of the image field theory, show that this latter theory can be very inaccurate for cases involving highly conducting substrates or large molecule-surface distances, consistent with previous observations for the case of flat surfaces.
\end{abstract}

\section{INTRODUCTION}

Surface-enhanced spectroscopy is a highly interdisciplinary area of research which has recently aroused immense interest from both chemists and physicists due to its various practical and fundamental aspects. ${ }^{1,2}$ Some typical examples of research interest involve possible enhanced photochemical processes, ${ }^{3,4}$ distortion of absorption line shapes for admolecules, ${ }^{5,6}$ and possible cooperative phenomena for an ensemble of admolecules. ${ }^{7}$ While some of this work may lead to practical applications such as laser-assisted heterogeneous catalysis, ${ }^{8}$ most of the fundamental goals of these investigations are aimed at the understanding of the mechanisms by which admolecules interact with substrate surfaces. ${ }^{9}$ Among these diverse phenomena, one common concern is the determination of the lifetimes of those molecules in the vicinity of the substrate surface. The reason for this is that the surface-induced decay rate for the admolecule will in many circumstances compete with the surfaceenhanced field, which will determine whether an ultimate enhancement is possible for processes such as fluorescence and other resonant absorption phenomena. ${ }^{1,3,4}$ This problem, namely, molecular lifetimes in the presence of a dielectric (often metallic) surface, has been investigated intensively for the last two decades, both theoretically ${ }^{10}$ and experimentally. ${ }^{11}$ By now it has become clear that the simplest and most appropriate theory to describe such phenomena under usual experimental conditions ${ }^{11}$ is the energy-transfer (ET) theory established chiefly by Chance, Prock, and Silbey ${ }^{10}$ (CPS) based on the Sommerfeld theory for a radiating dipole antenna above the surface of the earth. ${ }^{12}$ It is fair to say that, in most cases, as long as the molecule-surface distance is greater than a few angstroms so that the "quantum spreads" of both the surface electrons and admolecule can be neglected, ${ }^{13}$ this classical phenomenological (CP) approach has been found adequate. ${ }^{14}$ Furth- ermore, this theory (ET) leads to results identical with quantum-mechanical ones and reduces to the image theory (IT) in appropriate limits. ${ }^{10}$ Hence, the theoretical aspect of this problem seems to have been settled, at least for the case where perfect flatness is assumed for the surface boundary as in all these previous theories. ${ }^{10}$ For rough surfaces (which are more realistic in most experimental situations), however, there seems to be a lack of a complete theory which describes well such decay rates for the admolecules. Recently, prompted by the discrepancy observed between the CPS theory and the measurements of the decay rates for pyrazine molecules near certain noble-metal surfaces carried out by Rossetti and Brus (RB), ${ }^{15}$ the effects of surface roughness on such decay rates have been considered theoretically for both random $^{16}$ and periodic ${ }^{17}$ roughnesses as a possible explanation for the observed discrepancy in the RB experiments. Moreover, all these previous theories ${ }^{16,17}$ were based on the image-potential theory for a rough boundary established by Rahman and Maradudin. ${ }^{18}$ It has then been a wide belief ${ }^{1,10,19}$ that as long as the moleculer-surface separation $d$ is much shorter than the wavelengths $\lambda$ of the molecular emissions, which is true under most experimental conditions of interest $\left(d \leqslant 10^{2}\right.$ $\AA$ and $\left.\lambda \sim 10^{3} \AA\right),{ }^{15,20-22}$ such a static theory (IT) should yield results as good as the more exact dynamical theory (ET). However, it has been observed recently ${ }^{23}$ that the condition $d<<\lambda$ is not sufficient for IT to be valid. The exact comparison between ET and IT actually involves three distance scales, namely, $d, \lambda$, and the skin depth $\delta$ of the metal substrate. In addition to $d<<\lambda$, we must also require $d<<\delta$ for IT to be valid. In particular, for highly conducting substrates, such as silver $(\mathrm{Ag})$, it has been found that IT breaks down for $d \gtrsim 0.01 \lambda$ for the case of a flat surface. ${ }^{23}$ Roughly speaking, the physical origin for such a drawback of IT stems from the fact that the Helmholtz wave equation in a conducting media does not reduce to the static Poisson equation in the lim- 
it $\lambda \rightarrow \infty$ if the conductivity of the media becomes infinitely large.

It is therefore necessary to establish a dynamical theory (ET) for a complete description of the decay rates of admolecules at rough metallic surfaces. The present paper undertakes this task by a combination of the Sommerfeld antenna theory ${ }^{12}$ and the integral-equation formalism of Maxwell's equations for rough boundaries established chiefly by Maradudin and Mills ${ }^{24}$ and Agarwal. ${ }^{25}$ We shall present the theory in Sec. II, calculations for a simple configuration of the problem in Sec. III, and numerical studies in Sec. IV. For simplicity and for illustrative purposes, we shall always assume the molecular dipole to be located at $(0,0, d)$ and oriented perpendicular to the rough boundary. The generalization to other molecular orientations is straightforward (though tedious) by following the lines in the original CPS theory for flat boundary. Advantages and limitations of the present theory will be discussed further in the conclusion section.

\section{THEORY}

According to the CP approach, the admolecule is modeled as a point dipole $(\boldsymbol{\mu})$ described by the equation of motion for a damped harmonic oscillator. ${ }^{10}$ The molecular decay rates $(\gamma)$ in the presence of a substrate surface can be obtained in the form ${ }^{10,16,17}$

$$
\begin{aligned}
& \gamma_{F}=\gamma_{0}\left(1+\frac{3}{2} \frac{q}{k^{3}} \operatorname{Im} G^{F}\right), \\
& \gamma_{R}=\gamma_{0}\left(1+\frac{3}{2} \frac{q}{k^{3}} \operatorname{Im}\left(G^{F}+G^{R}\right)\right),
\end{aligned}
$$

where $q$ is the quantum yield of the emitting state, $k$ is the emission wave number, and the indices $R, F$, and 0 stand for cases with a rough surface, flat surface, and free molecule, respectively. The $G$ functions of Eqs. (1) and (2) are defined as the reflected $\left(\mathbf{E}^{r}\right)$ from the surface boundary acting on the molecular dipole per unit dipole moment as a function of the emission frequency,

$$
G(\omega)=\frac{E^{r}(\omega)}{\mu} .
$$

Note that in writing Eq. (2), we have followed previous approaches ${ }^{16,17}$ by separating the contributions to the surface-induced decay rates from the flat boundary and from the roughness, respectively. To exhibit more clearly the effect due to the surface roughness, it is useful to define the ratio

$$
R \equiv \frac{\gamma_{R}}{\gamma_{F}}=1+\frac{\frac{3}{2} \frac{q}{k^{3}} \operatorname{Im} G^{R}}{1+\frac{3}{2} \frac{q}{k^{3}} \operatorname{Im} G^{F}} .
$$

The remaining task in this theory is to calculate the $G(\omega)$ functions. In general, within the CP approach, there are two ways of doing this. In the exact treatment (ET), one has to solve the full set of Maxwell's equations (ME) (or equivalently, the Helmholtz wave equation) by regarding the dipole emission as the incident (source) field $\mathbf{E}^{(0)}$ and matching boundary conditions at the surface. Thus for the case of a flat surface, Chance, Prock, and Silbey ${ }^{10}$ solved this problem by applying Sommerfeld's theory for a radiating dipole antenna above the earth's surface. ${ }^{26}$ As an example, for $\mu$ located at $(0,0, d)$ and oriented perpendicular to the surface, the CPS theory gives ${ }^{10}$

$$
G_{\mathrm{ET}}^{F}(\omega)=-k^{3} \int_{0}^{\infty} d u R e^{-2 l_{1} \bar{d} \frac{u^{3}}{l_{1}}},
$$

where $\quad \omega=c k, \quad \bar{d}=k d, \quad R=\left(l_{2}-\epsilon l_{1}\right) /\left(l_{2}+\epsilon l_{1}\right), \quad l_{1}=$ $-i\left(1-u^{2}\right)^{1 / 2}, \quad l_{2}=-i\left(\epsilon-u^{2}\right)^{1 / 2}, \quad$ and $\epsilon(\omega)=\epsilon_{1}(\omega)$ $+i \epsilon_{2}(\omega)$ is the bulk dielectric constant of the substrate material.

The other method of calculating the $G$ functions would be the IT. This theory, which is approximate but much simpler, regards $\mathbf{E}^{r}$ as given by the image field, which is obtained from solving the electrostatic Poisson equation by matching the appropriate boundary conditions. ${ }^{18}$ Thus for the above example, one obtains

$$
G_{\mathrm{IT}}^{F}(\omega)=\frac{k^{3}}{4 \bar{d}^{3}} \frac{\epsilon-1}{\epsilon+1} .
$$

While both theories (ET and IT) have been established for a flat surface boundary, to our knowledge only IT is available for situations where surface roughness cannot be neglected. ${ }^{16,17}$ However, in a previous publication, ${ }^{23}$ we have pointed out that IT can give very unreliable results for highly conducting substrates even in the limit $d<<\lambda$. Hence in this section we shall attempt to establish a dynamical theory (ET) for rough surfaces by calculating $G_{\mathrm{ET}}^{R}(\omega)$ below.

The calculation of reflected fields $\mathbf{E}^{r}$ from rough surfaces is a long-existing problem beginning with the classic papers of Rayleigh and Fano. ${ }^{27}$ By now it has become clear that there are two general ways of approaching the problem: direct boundary matching of the field quantities $^{28-30}$ or application of the integral-equation formalism. $^{24,25}$ In the former approach, either the vector potentials ${ }^{28}$ or the fields ${ }^{29,30}$ are matched across the boundary with a profile function $z=\zeta(x, y)$, and the solutions are obtained usually to the lowest order in $\zeta$ by a perturbation method. ${ }^{28,30}$ Although a more general solution has been obtained by Toigo et al. ${ }^{29}$ which in principle (though tedious) can give expressions for the field to any order in $\zeta$, the Raleigh hypothesis ${ }^{27}$ (which is known to guarantee convergence only for small- $\zeta$ cases) has nevertheless been assumed in all these formalisms, ${ }^{28-31}$ so that they are of limited applicability in this sense. On the other hand, the integral-equation approach usually starts by avoiding direct matching of the field quantities across the boundary $z=\zeta(x, y)$ by converting the Maxwell-Helmholtz equation to an integral equation by means of the Green's-function method ${ }^{24}$ or the application of the extinction theorem. ${ }^{32}$ The Green's-function method of Maradudin and Mills ${ }^{24}$ (MM) has been criticized by other researchers ${ }^{28}$ (who have followed the direct-matching approach) as being too crude with their perturbative approximations which led to final solutions 
inconsistent with those obtained by these later researchers. However, the correct reason which leads to such disagreements between these two approaches was only pointed out later by Agarwal, ${ }^{25}$ who proved that the MM formalism is actually correct and consistent with all other approaches ${ }^{28,30}$ (including the extinction theorem approach $^{32}$ ), except that MM had not treated the discontinuity of the field quantities across the plane $z=0$ correctly. With a remedial recipe, Agarwal has shown that the MM formalism can actually lead to the correct results. Hence we shall adopt the MM theory ${ }^{24}$ in this present investigation to calculate $G_{\mathrm{ET}}^{R}(\omega)$ and shall discuss the advantages and limits of this formalism in Sec. V. ${ }^{33}$

The main idea of MM's theory is to regard the surface roughness $\zeta$ as a source to the homogeneous Hemholtz equation for the case of a perfectly flat boundary. Thus, by expanding the dielectric constant in the form

$$
\begin{aligned}
\epsilon(z, \omega)= & \epsilon_{0}(z, \omega)+[\epsilon(\omega)-1] \xi(x, y) \delta(z) \\
& -\frac{1}{2}[\epsilon(\omega)-1] \zeta^{2}(x, y) \delta^{\prime}(z)+O\left(\zeta^{3}\right),
\end{aligned}
$$

with

$$
\epsilon_{0}(z, \omega)=\left\{\begin{array}{l}
1, \quad z>0 \\
\epsilon(\omega), \quad z<0,
\end{array}\right.
$$

the Helmholtz equation for the $\mu$ th component of the total electric field can be expressed in the form of an integral equation, and to lowest order in $\zeta$ one has ${ }^{24}$

$$
\begin{aligned}
E_{\mu}(\mathbf{r}, \omega)=E_{\mu}^{0}(\mathbf{r}, \omega)- & \frac{k^{2}}{4 \pi}[\epsilon(\omega)-1] \\
& \times \int d^{3} r^{\prime} D_{\mu \nu}\left(\mathbf{r}, \mathbf{r}^{\prime} ; \omega\right) \\
& \times \zeta\left(x^{\prime}, y^{\prime}\right) \delta\left(z^{\prime}\right) E_{v}\left(\mathbf{r}^{\prime} ; \omega\right),
\end{aligned}
$$

where $E_{\mu}^{0}$ is the total field for perfectly flat boundaries, $D_{\mu \nu}$ is the Green's tensor ("propagator") for the corresponding wave equation, and Einstein's summation convention has been adopted. Following MM's approach, by invoking the "Born-type" approximation and by introducing the two-dimensional Fourier transforms ${ }^{34}$

$D_{\mu \nu}\left(\mathbf{r}, \mathbf{r}^{\prime} ; \omega\right)=\int \frac{d^{2} k_{\|}}{(2 \pi)^{2}} e^{i \mathbf{k}_{\|} \cdot\left(\mathbf{r}_{\|}-\mathbf{r}_{\|}^{\prime}\right)} d_{\mu \nu}\left(\mathbf{k}_{\|} \omega \mid z z^{\prime}\right)$, $\zeta\left(\mathbf{r}_{\|}\right)=\int \frac{d^{2} k_{\|}}{(2 \pi)^{2}} e^{i \mathbf{k}_{\|} \cdot \mathbf{r}_{\|}} \hat{\zeta}\left(\mathbf{k}_{\|}\right)$,

$E_{\mu}^{(0)}(\mathbf{r} ; \omega)=e^{i \mathbf{k}_{\|}^{(0)} \cdot \mathbf{r}_{\|}} E_{\mu}^{(0)}\left(\mathbf{k}_{\|}^{(0)} \omega \mid z\right)$,

with $\mathbf{r}_{\|}=(x, y, 0)$ and $\mathbf{k}_{\|}=\left(k_{x}, k_{y}, 0\right)$, we can finally obtain the total reflected field $\left(\mathbf{E}^{r}\right)$ in the presence of surface roughness in the form

$$
E_{\mu}^{r}=E_{\mu}^{r F}+E_{\mu}^{r R}
$$

where $F$ and $R$ stand for the contributions from the flat boundary and the roughness, respectively. According to MM, we have to order $\zeta$ (Ref. 35)

$$
\begin{gathered}
E_{\mu}^{r R}(\mathbf{r} ; \omega)=-\frac{k^{2}}{16 \pi^{3}}[\epsilon(\omega)-1] \\
\times \int d^{2} k_{\|} e^{i \mathbf{k}_{\|} \cdot \mathbf{r}_{\|}} \hat{\zeta}\left(\mathbf{k}_{\|}-\mathbf{k}_{\|}^{(0)}\right) \\
\times \int d z^{\prime} d_{\mu v}\left(\mathbf{k}_{\|} \omega \mid z z^{\prime}\right) \\
\times \delta\left(z^{\prime}\right) E_{v}^{(0)}\left(\mathbf{k}_{\|}^{(0)} \omega \mid z^{\prime}\right) .
\end{gathered}
$$

We notice already from Eqs. (13) and (14) that one good feature of this theory is that $E_{\mu}^{r}$ can be expressed completely in terms of the flat-surface solutions (with $E_{\mu}^{r F}=E_{\mu}^{(0)}-E_{\mu}^{\text {in }}, E_{\mu}^{\text {in }}$ being the incident field), which are in general easily attainable. Thus, towards our ultimate goal of the calculation of $G_{\mathrm{ET}}^{R}(\omega) \equiv E^{r R} / \mu$, we can just insert the solution for $E_{v}^{(0)}$ from the CPS theory ${ }^{10}$ into Eq. (14) since all the Green's tensors $d_{\mu \nu}$ are already given in $\mathbf{M M}^{24}$

Before going to specific case studies, let us also give the result for $E_{\mu}^{r R}$ to order $\zeta^{2}$, which is the lowest nonvanishing order when small (Gaussian-distributed) random roughness is present. ${ }^{16}$ One must be very careful and consistent in working out the $\mathrm{MM}$ theory to this order. In particular, one has to collect terms from the second-order Born-type approximation to order $\zeta$ in $E_{\mu}(\mathbf{r} ; \omega)$ in Eq. (9) together with those from the firstorder Born-type approximation to order $\zeta^{2}$ in the original, exact integral equation for $E_{\mu}(\mathbf{r} ; \omega)$. With some manipulation, we finally obtain ${ }^{35}$ to order $\zeta^{2}$

$$
\begin{aligned}
& E_{\mu}^{r R}(\mathbf{r} ; \omega)=-\frac{k^{2}}{16 \pi^{3}}[\epsilon(\omega)-1]\left[\int d ^ { 2 } k _ { \| } e ^ { i \mathbf { k } _ { \| } \cdot \mathbf { r } _ { \| } } \left[\hat{\xi}\left(\mathbf{k}_{\|}-\mathbf{k}_{\|}^{(0)}\right) \int d z^{\prime} d_{\mu v}\left(\mathbf{k}_{\|} \omega \mid z z^{\prime}\right) \delta\left(z^{\prime}\right) E_{v}^{(0)}\left(\mathbf{k}_{\|}^{(0)} \omega \mid z^{\prime}\right)\right.\right. \\
&+\frac{1}{8 \pi^{2}} \int d^{2} k_{\|}^{\prime} \hat{\xi}\left(\mathbf{k}_{\|}^{\prime}\right) \hat{\xi}\left(\mathbf{k}_{\|}-\mathbf{k}_{\|}^{\prime}-\mathbf{k}_{\|}^{(0)}\right) \\
& \times \int d z^{\prime} \frac{\partial}{\partial z^{\prime}}\left[d_{\mu v}\left(\mathbf{k}_{\|} \omega \mid z z^{\prime}\right) E_{v}^{(0)}\left(\mathbf{k}_{\|}^{(0)} \omega \mid z^{\prime}\right)\right] \delta\left(z^{\prime}\right) \\
&-\frac{k^{2}}{16 \pi^{5}}[\epsilon(\omega)-1] \int d^{2} k_{\|}^{\prime} \hat{\xi}\left(\mathbf{k}_{\|}\right) \hat{\xi}\left(\mathbf{k}_{\|}^{\prime}-\mathbf{k}_{\|}^{(0)}\right) e^{i \mathbf{k}_{\|}^{\prime} \cdot \mathbf{r}_{\|} \int d z^{\prime} d_{\mu v}\left(\mathbf{k}_{\|} \omega \mid z z^{\prime}\right) \delta\left(z^{\prime}\right)} \\
&\left.\times \int d z^{\prime \prime} d_{\mu v}\left(\mathbf{k}_{\|}^{\prime} \omega \mid z z^{\prime \prime}\right) \delta\left(z^{\prime \prime}\right) E_{v}^{(0)}\left(\mathbf{k}_{\|}^{(0)} \omega \mid z^{\prime \prime}\right)\right]
\end{aligned}
$$




\section{CALCULATION}

Here we want to illustrate the dynamical theory (ET) established in Sec. II by considering the simplest case that one can have, namely, a perpendicular dipole $(\boldsymbol{\mu})$ located at $(0,0, d)$ above a shallow sinusoidal grating surface $\zeta(x, y)=\zeta_{0} e^{i Q x}$. Previously, we have obtained to or$\operatorname{der} \zeta$ the $G$ function for this case via the image method (IT), which can be expressed as ${ }^{17}$

$$
\begin{aligned}
G_{I T}^{R}= & \frac{4 \zeta_{0}}{\pi} \frac{\epsilon-1}{(\epsilon+1)^{2}} \\
& \times \int_{0}^{\infty} d u \int_{0}^{\infty} d v(\epsilon f g+h) \exp [-(f+g) d],
\end{aligned}
$$

where $f, g$, and $h$ are functions of $u$ and $v$, given by

$$
\begin{aligned}
& f(u, v)=\left[\left[u+\frac{Q}{2}\right]^{2}+v^{2}\right]^{1 / 2}, \\
& g(u, v)=\left[\left[u-\frac{Q}{2}\right]^{2}+v^{2}\right]^{1 / 2}, \\
& h(u, v)=u^{2}+v^{2}-\frac{Q^{2}}{4} .
\end{aligned}
$$

In the following, we shall calculate $G_{\mathrm{ET}}^{R}$ for this case according to Eq. (14) and then compare with the results obtained from Eq. (16).

First, let us start by recalling the fields obtained in the CPS theory for this case. For a flat surface boundary at $\mathbf{r}=(0,0, z)$, CPS theory gives ${ }^{10}$

$$
\begin{aligned}
& \mathbf{E}^{\mathrm{in}}(z ; \omega)=\widehat{\mathbf{e}}_{z} \mu k^{3} \int_{0}^{\infty} d u \frac{u^{3}}{l_{1}} e^{l_{1}(\bar{z}-\bar{d})}, \\
& \mathbf{E}^{r F}(z ; \omega)=-\widehat{\mathbf{e}}_{z} \mu k^{3} \int_{0}^{\infty} d u R \frac{u^{3}}{l_{1}} e^{-l_{1}(\bar{z}+\bar{d})}, \\
& \mathbf{E}^{t F}(z ; \omega)=\widehat{\mathbf{e}}_{z} \mu k^{3} \int_{0}^{\infty} d u \frac{1}{\epsilon}(1-R) \frac{u^{3}}{l_{1}} e^{l_{2} \bar{z}-l_{1} \bar{d}},
\end{aligned}
$$

with $\bar{z}=k z$ and other quantities defined as in Eq. (5). Equations (20)-(22) represent the incident, reflected, and transmitted fields, respectively. To simplify our application of these results to calculate Eq. (14), we assume further that $\mathbf{k}_{\|}^{(0)} \cong 0$, i.e., only the wave normally incident on the surface is reflected back at the location of the source dipole. Obviously, this approximation is good only when the roughness is small. ${ }^{36}$ Granting this approximation, we then have for $E_{v}^{(0)}$ within Eq. (14)

$$
E_{v}^{(0)}\left(\mathbf{k}_{\|}^{(0)} \omega \mid z^{\prime}\right) \cong E_{v}^{(0)}\left(z^{\prime}, \omega\right) \delta_{v z},
$$

and since $\mathbf{E}^{(0)} \equiv \mathbf{E}^{\text {in }}+\mathbf{E}^{r F}$, from Eqs. (20) and (21) we obtain (for $z^{\prime}>0$ )

$$
\begin{aligned}
& E_{v}^{(0)}\left(\mathbf{k}_{\|}^{(0)} \omega \mid z^{\prime}\right) \\
& \quad \cong \mu k^{3} \int_{0}^{\infty} d u \frac{u^{3}}{l_{1}}\left(e^{l_{1}\left(\bar{z}^{\prime}-\bar{d}\right)}-\mathrm{Re}^{-l_{1}\left(\bar{z}^{\prime}+\bar{d}\right)}\right) \delta_{v z} .
\end{aligned}
$$

Furthermore, using the Appendix of the MM paper, ${ }^{24}$ we obtain

$$
d_{z z}\left(\mathbf{k}_{\|} \omega \mid z z^{\prime}\right)=\left\{\begin{array}{l}
\frac{2 \pi i k_{\|}^{2}}{k k_{2}}\left[\frac{k_{1}+\epsilon(\omega) k_{2}}{k_{1}-\epsilon(\omega) k_{2}} e^{i k_{2}\left(z+z^{\prime}\right)}-e^{i k_{2}\left|z-z^{\prime}\right|}\right]+\frac{4 \pi}{k^{2}} \delta\left(z-z^{\prime}\right) \text { for } z>0, z^{\prime}>0 \\
\frac{4 \pi i k_{\|}^{2}}{k^{2}} \frac{1}{k_{1}-\epsilon(\omega) k_{2}} e^{i k_{2} z+i k_{1} z^{\prime}} \text { for } z>0, z^{\prime}<0,
\end{array}\right.
$$

where $k=\omega / c, \mathbf{k}_{\|}=\left(k_{x}, k_{y}, 0\right)$, and

$$
\begin{aligned}
& k_{1}=-\left[\epsilon(\omega) k^{2}-k_{\|}^{2}\right]^{1 / 2}, \\
& k_{2}= \begin{cases}\left(k^{2}-k_{\|}^{2}\right)^{1 / 2}, & k^{2}>k_{\|}^{2} \\
i\left(k_{\|}^{2}-k^{2}\right)^{1 / 2}, & k^{2}<k_{\|}^{2} .\end{cases}
\end{aligned}
$$

With (23) and setting $\mathbf{k}_{\|}^{(0)} \cong 0$ in Eq. (14), we finally obtain (with $\mathbf{r}_{\|}=0$ )

$$
\begin{aligned}
& E_{z}^{r R}(z ; \omega)=- \frac{k^{2}}{16 \pi^{3}}[\epsilon(\omega)-1] \\
& \times \int d^{2} k_{\|} \hat{\xi}\left(\mathbf{k}_{\|}\right) \int d z^{\prime} d_{z z}\left(\mathbf{k}_{\|} \omega \mid z z^{\prime}\right) \\
& \\
& \times E_{z}^{(0)}\left(z^{\prime}, \omega\right) \delta\left(z^{\prime}\right),
\end{aligned}
$$

where $d_{z z}$ is given by Eqs. (24)-(26).

Let us first evaluate the integral involving $z^{\prime}$ by taking care of the discontinuity of the function $d_{z z} E_{z}^{(0)}$ at $z^{\prime}=0$. Following MM (Ref. 24) and Agarwal's ${ }^{25}$ prescription, we finally obtain the following equivalent expressions:

$$
\begin{aligned}
\int d z^{\prime} d_{z z}\left(\mathbf{k}_{\|} \omega \mid z z^{\prime}\right) & E_{z}^{(0)}\left(z^{\prime}, \omega\right) \delta\left(z^{\prime}\right) \\
= & d_{z z}\left(\mathbf{k}_{\|} \omega \mid z 0_{+}\right) E_{z}^{(0)}\left(0_{-}, \omega\right) \\
= & \frac{1}{\epsilon(\omega)} d_{z z}\left(\mathbf{k}_{\|} \omega \mid z 0_{+}\right) E_{z}^{(0)}\left(0_{+}, \omega\right) \\
= & \epsilon(\omega) d_{z z}\left(\mathbf{k}_{\|} \omega \mid z 0_{-}\right) E_{z}^{(0)}\left(0_{-}, \omega\right) .
\end{aligned}
$$

Using any one of the results of Eq. (28) and with the help of Eqs. (22) or (23) and Eq. (24), we finally obtain a unique expression for Eq. (27) at $z=d$ given by

$$
\begin{aligned}
E_{z}^{r R}(d ; \omega)=-i \mu k^{3} \frac{\epsilon-1}{4 \pi^{2}} \int d^{2} k_{\|} \hat{\zeta}\left(\mathbf{k}_{\|}\right) \frac{k_{\|}^{2} e^{i k_{2} d}}{k_{1}-\epsilon k_{2}} \\
\times \int_{0}^{\infty} d u(1-R) \frac{u^{3}}{l_{1}} e^{-l_{1} \bar{d}} .
\end{aligned}
$$

With the Fourier transform for the shallow sinusoidal 
grating profile given by

$$
\hat{\zeta}\left(\mathbf{k}_{\|}\right)=(2 \pi)^{2} \zeta_{0} \delta\left(\mathbf{Q}-\mathbf{k}_{\|}\right),
$$

where

$$
\mathbf{Q}=Q \widehat{\mathbf{e}}_{x},
$$

Eq. (29) finally yields

$$
\begin{aligned}
G_{\mathrm{ET}}^{R}=\frac{E_{z}^{r R}}{\mu}=- & i k^{3} \xi_{0}(\epsilon-1) \frac{Q^{2} e^{i k_{2} d}}{k_{1}-\epsilon k_{2}} \\
& \times \int_{0}^{\infty} d u(1-R) \frac{u^{3}}{l_{1}} e^{-l_{1} \bar{d}},
\end{aligned}
$$

where

$$
\begin{aligned}
& k_{1}=-\left[\epsilon(\omega) k^{2}-Q^{2}\right]^{1 / 2} \\
& k_{2}= \begin{cases}\left(k^{2}-Q^{2}\right)^{1 / 2}, & k^{2}>Q^{2} \\
i\left(Q^{2}-k^{2}\right)^{1 / 2}, & k^{2}<Q^{2}\end{cases}
\end{aligned}
$$

Combining Eqs. (5) and (32) with Eq. (2), we have a complete dynamical description (ET) of molecular decay rates at shallow sinusoidal grating surfaces. On the other hand, Eqs. (6) and (16) together provide the static calculations (IT) for the same problem from Eq. (2). ${ }^{17}$

\section{NUMERICAL RESULTS AND DISCUSSIONS}

We have compared the two theories by computing both the quantities $\operatorname{Im} G^{R}$ and the ratio $R$ in Eq. (4) via the two theories (ET and IT) for different grating parameters and molecular emission frequencies. ${ }^{37}$ Figure 1 shows the comparison of $\operatorname{Im} G_{\mathrm{IT}}^{R}$ and $\operatorname{Im} G_{\mathrm{ET}}^{R}$ according to Eqs. (16) and (32), respectively, for a shallow Ag grating $\left(\zeta_{0}=0.7 \AA, Q=0.01 \AA^{-1}\right)$ at $\omega=2.5 \mathrm{eV}$, from which one can see that IT gives correct results only for small values of $d$ and lies consistently below ET for larger $d$ values, a behavior consistent with that observed previously for flat surfaces. ${ }^{23}$ Figure 2 shows the same comparison for a $\mathrm{Ni}$ surface $(\omega=3.3 \mathrm{eV})$, which exhibits similar discrepancies between the two theories. Although $\mathrm{Ni}$ is a less conducting material than Ag, the anticipated better agreement between the two theories for $\mathrm{Ni}$ is manifested only slightly in the short-distance regime (e.g., the deviation between ET and IT at $d=20 \AA$ is about $28 \%$ for $\mathrm{Ni}$ and $36 \%$ for $\mathrm{Ag}$ ), in contrast to the case for flat surfaces where such comparison is more sensitive to the conductivity of the substrate surface. ${ }^{23}$ As discussed before, the physical origin for the "largedistance discrepancies" is that IT includes only nonradiative energy transfer, whereas ET includes both radiative and nonradiative energy transfers, and hence the induced molecular damping is more serious as compared to IT at large molecule-surface separation. ${ }^{23}$ Figure 3 shows a calculation of the ratio $\mathcal{R}$ in Eq. (4) as a function of $d$ according to ET [Eqs. (5) and (32)] for different emission frequencies and grating parameters for a $\mathrm{Ag}$ surface. As before, we have assumed $q \cong 1 .^{10,17}$ It is of interest to observe that while all graphs show the disappearance of the roughness effects at large values of $d$, $R \rightarrow 1$ much faster for the case of a deeper grating

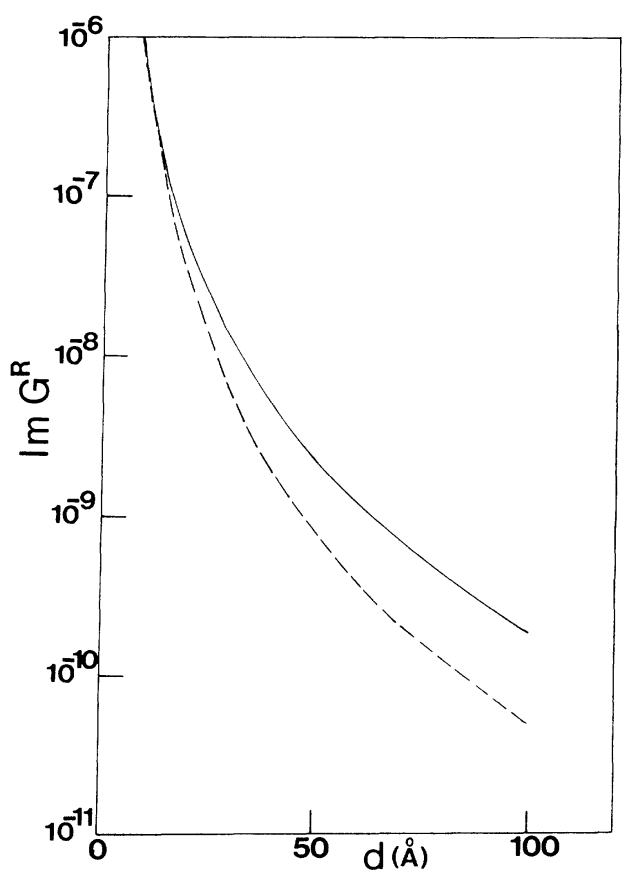

FIG. 1. Comparison between the energy-transfer theory (ET, solid curve) and the image theory (IT, dashed curve) for a $\mathrm{Ag}$ grating substrate $\left(\zeta_{0}=0.7 \AA, Q=0.01 \AA^{-1}\right)$ at $\omega=2.5 \mathrm{eV}$ for a range of molecule-surface distances $d$. The unit of $G$ is in $\AA^{-3}$.

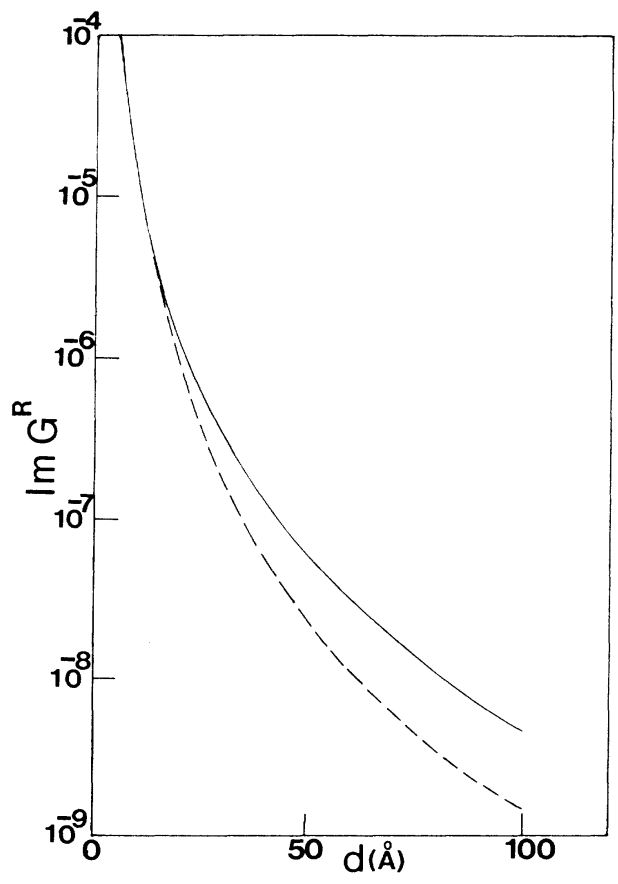

FIG. 2. Same as Fig. 1, but for a Ni grating at $\omega=3.3 \mathrm{eV}$. 


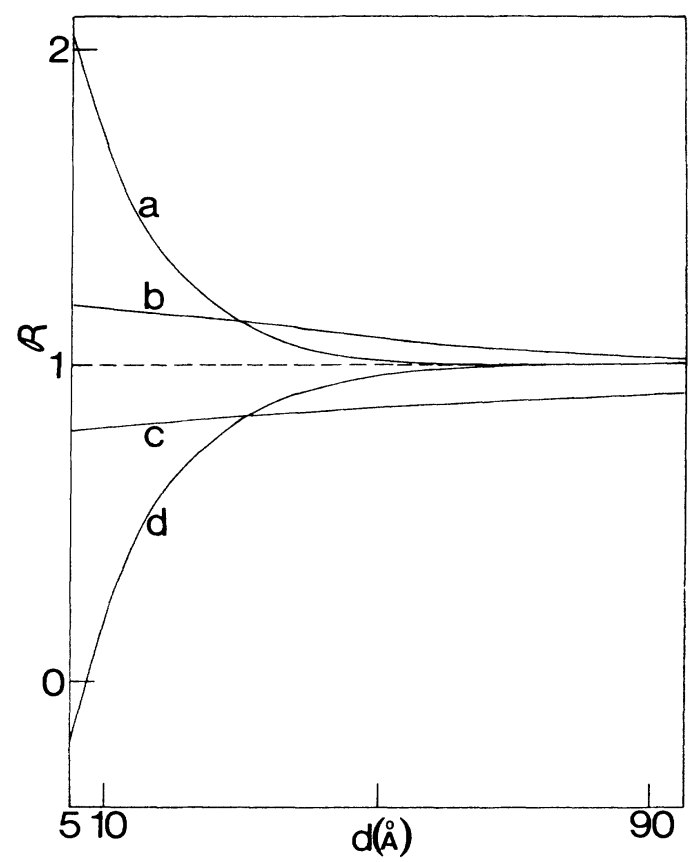

FIG. 3. Comparison of the molecular decay rates as given by ET at the rough $\mathrm{Ag}$ surface as in Fig. 1 with those for a flat surface $(\mathcal{R})$ vs $d$ for different emitting frequencies and grating period. Curve $a$ : $\omega=2.5 \mathrm{eV}, Q=0.08 \AA^{-1}$. Curve $b: \omega=2.5$ eV, $Q=0.01 \AA^{-1}$. Curve $c: \omega=3.74 \mathrm{eV}, Q=0.01 \AA^{-1}$. Curve $d: \omega=3.74 \mathrm{eV}, Q=0.08 \AA^{-1}$.

$\left(Q=0.08 \AA^{-1}\right)$. Furthermore, for $\omega=3.74 \mathrm{eV} \cong \omega_{\mathrm{sp}}$ [the surface plasmon (SP) resonance frequency], we observe that roughness can cause diminution of the decay rate from its flat surface values $(\mathcal{R}<1)$, consistent with similar results obtained previously using IT. ${ }^{17}$ For a more comprehensive picture, we show in Fig. 4 a comparison between ET and IT for values of $\mathcal{R}$ versus $d$ for a Ag surface from which we can see that at close distances the discrepancy between $\operatorname{Im} G_{\mathrm{ET}}^{F}$ and $\operatorname{Im} G_{\mathrm{IT}}^{F}$ predominates, so that $\mathscr{R}$ for IT lies above that for ET. A calculation for the Ni surface shows similar behavior. It is consistent to conclude that due to the inclusion of radiative transfer, the effect of the roughness survives at larger molecule-surface distances in ET as compared to IT. Finally, in Fig. 5 we show the variation of $\gamma_{R} / \gamma_{0}$ [Eq. (2)] with emission frequencies $\omega$ at fixed distance $(d=10 \AA$ ) for the same $\mathrm{Ag}$ grating as in Fig. 1. Here we observe that while at such close distances IT should be reasonably accurate as compared to ET, the two theories show the largest deviation from each other in the neighborhood of the SP resonance frequency. This is due to the fact that from Eqs. (16) and (32) it is obvious that the resonance factors such as $\epsilon+1$ in IT and $k_{1}-\epsilon k_{2}$ in ET are very different from each other [the same different resonance structure occurs for $G^{F}$ as can be seen from Eqs. (5) and (6)]. Hence we should expect that IT should not be trustworthy at $\omega \cong \omega_{\mathrm{SP}}$, no matter how close the molecule is from the surface.

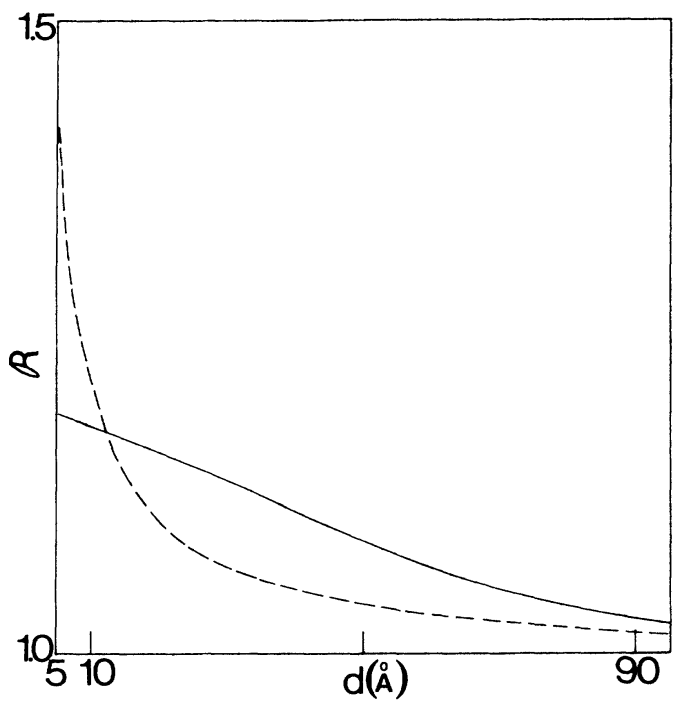

FIG. 4. Comparison between ET and IT for the ratio $R$ vs $d$. Other conditions are the same as in Fig. 1.

\section{CONCLUSION}

As extensions of our previous work, ${ }^{17,23}$ we have in this paper established a dynamical theory (ET) for the classical decay rates of molecules at rough metallic surfaces. In particular, details for a perpendicular dipole above a shallow sinusoidal grating have been worked out and compared with the previous results obtained using the image theory. ${ }^{17}$ Once again, we have observed the role played by the substrate conductivity (though to a comparatively minor extent) as occurring in the case of flat surfaces. ${ }^{23}$ Presumably, if one applies the result ob-

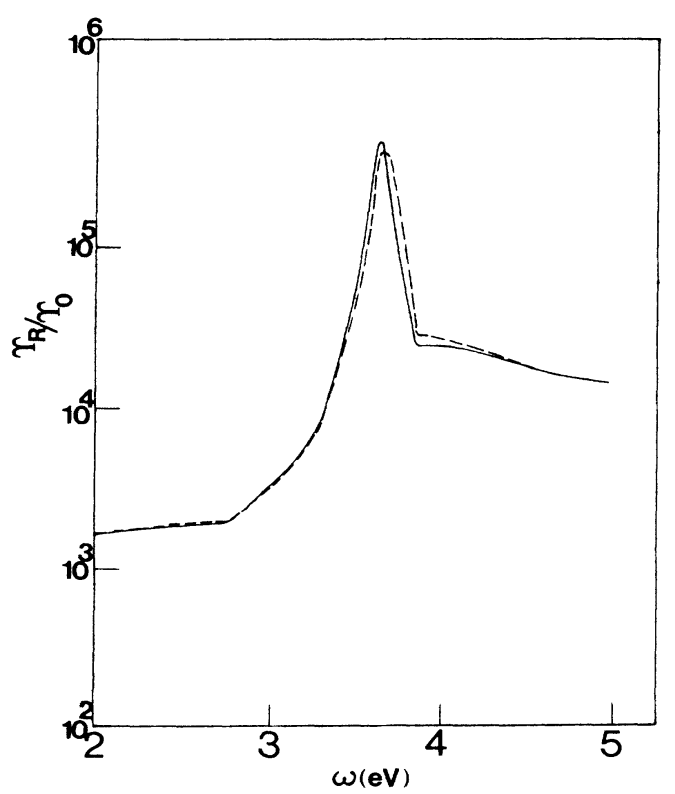

FIG. 5. Comparison between ET and IT for the ratio $\gamma_{R} / \gamma_{0}$ vs $\omega$ at fixed $d=10 \AA$ for the same Ag grating as in Fig. 1 . 
tained in Eq. (15) to the case of Gaussian-distributed random roughness for a perpendicularly oriented dipole, the results for $\gamma_{R}$ then obtained should show similar comparisons with those obtained before using IT for this case by Arias, Aravind, and Metiu. ${ }^{16}$ We have pointed out that more complicated configurations (e.g., molecules oriented parallel to the surface) can also be treated by the combination of the CPS (Ref. 10) and MM (Ref. 24) theories along similar lines as developed in this paper. We want to give below some further comments on the MM theory ${ }^{24}$ which we have applied.

As mentioned in Sec. II, all these past theories ${ }^{24,25,27-32}$ for the calculation of reflected fields from rough surfaces have advantages and disadvantages. Here we just want to summarize the advantages and the drawbacks of the MM theory. Our results in Eqs. (14), (15), (29), and (32) will enjoy (suffer) the same advantages (drawbacks) as in the MM theory. First we want to restate that the most serious limitations of this theory is the use of the two-dimensional Fourier transform for the Green's tensor $D_{\mu \nu}$ in Eq. (10), which assumes translational invariance along the surface. This use of the socalled "bare propagator" has also been commented on by some other investigators ${ }^{33}$ and has limited the accuracy of the theory to only lowest orders in the surface roughness $\zeta(x, y)$. Nevertheless, it is exactly this approximation which renders the MM formalism capable of avoiding direct matching of the boundary conditions for the field quantities across the rough surface, which is a complicated task and for which explicit analytic results have been obtained only to order $\zeta .^{28,30}$ Expressions to order $\zeta^{2}$ will be very difficult (if not formidable) to obtain via the direct-matching method. However, within the MM theory, as we have seen in Sec. II, one can obtain systematically (though tediously) results of higher order in $\zeta$ as long as one is careful in collecting terms of the same order in both the roughness and the kind of "Born-type" approximation which has been applied. Since this approach has been shown by Agarwal ${ }^{25}$ explicitly to give identical results obtained by all other methods (at least to order $\zeta$ ), we have therefore adopted the MM approach in our present theory. Furthermore, the MM theory is a vector theory, which thus has advantages over ordinary scalar theories. ${ }^{31}$ It is possible that some very recent formalism using path-integral approaches by Gersten and Nitzan ${ }^{38}$ to the same problem may lead to more handy and accurate numerical treatments of the calculations of the decay rates, and this will be left as a future investigation. In addition, the assumption that $\mathbf{k}_{\|}^{(0)} \cong 0$ restricts our result in Eq. (32) to be accurate only for very shallow gratings, although this restriction has nothing to do with the MM theory. In principle, one can carry out a more complicated analysis of the different possible reflected rays back at the molecular site taking into consideration the interference effects. However, for those numerical examples which we have been analyzing $\left(\zeta_{0} Q \lesssim 0.006\right)$, the approximation $\mathbf{k}_{\|}^{(0)} \cong 0$ should be very accurate. ${ }^{36}$

Finally, we want to indicate that in the past few years there have been a lot of theoretical and experimental activities dealing with the comparison between the CPS theory (ET), image theory (IT) and the quantummechanical models due to Persson and co-workers. ${ }^{39}$ The latest experimental study (to our knowledge) is the one published Alivisatos, Waldek, and Harris, ${ }^{14}$ which seems to give support to the Persson theory as opposed to the classical CPS theory, even at large moleculesurface distances. This is quite surprising since Persson's original theory, ${ }^{39}$ when compared to the static image theory, shows that the discrepancy is again related to the conductivity of the substrate, the same origin for the discrepancy between ET and IT found in our recent work. ${ }^{23}$ Furthermore, as admitted by Alivisatos, Waldek, and Harris in the conclusion of their paper, ${ }^{14}$ it is in fact difficult for them from their experimental data to distinguish between the CPS and the Persson theories. In addition, surface roughness may very likely play a role in the comparison between these experiments and theories. Thus, it would be very interesting to compare and clarify these three different theories, namely, ET, IT, and the Persson theory (taking into consideration the effects of surface roughness) for different molecular emission frequencies and substrate conductivities. Work is in progress in this direction in our group.

\section{ACKNOWLEDGMENTS}

We thank Mr. Y. S. Kim for discussions and help in computations. This research was supported by the Air Force Office of Scientific Research (AFSC), United States Air Force, under Contract F49620-86-C-0009, and the Office of Naval Research.
${ }^{1}$ H. Metiu, Prog. Surf. Sci. 17, 153 (1984).

${ }^{2}$ M. Moskovits, Rev. Mod. Phys. 57, 783 (1985).

${ }^{3}$ J. I. Gersten and A. Nitzan, Surf. Sci. 158, 165 (1985), and references therein.

${ }^{4}$ P. T. Leung and T. F. George, J. Chem. Phys. 85, 4729 (1986).

${ }^{5}$ D. C. Langreth, Phys. Rev. Lett. 54, 126 (1985).

${ }^{6}$ P. T. Leung and T. F. George, Chem. Phys. Lett. 134, 375 (1987).

${ }^{7}$ K. C. Liu and T. F. George, Phys. Rev. B 32, 3622 (1985).

${ }^{8}$ For heterogeneous catalysis, see, e.g., R. H. Williams and I. T.
McGovern, in The Chemistry and Physics of Solid Surfaces and Heterogeneous Catalysis, edited by D. A. King and D. P. Woodruff (Elsevier, New York, 1984) Vol. 3B.

${ }^{9}$ Some familiar mechanisms include electron-hole pair creation, surface-plasmon excitation, surface-phonon, polaron, and other elementary excitations.

${ }^{10}$ See, e.g., R. R. Chance, A. Prock, and R. Silbey, Adv. Chem. Phys. 37, 1 (1978) and references therein. This article contains a very comprehensive review of the literature.

${ }^{11}$ For earlier experimental efforts, see, e.g., K. H. Drexhage, in 
Progress in Optics, edited by E. Wolf (North-Holland, Amsterdam, 1974), Vol. XII, p. 165ff. More recent experiments are mentioned individually in the text.

${ }^{12}$ A. Sommerfeld, Ann. Phys. (Leipzig) 28, 665 (1909); 81, 1135 (1926); Partial Differential Equations of Physics (Academic, New York, 1949).

${ }^{13}$ G. E. Korzeniewski, T. Maniv, and H. Metiu, J. Chem. Phys. 76, 1564 (1982).

${ }^{14}$ Although there has been some recent controversy as to the validity of the CPS theory [see A. P. Alivisatos, D. H. Waldeck, and C. B. Harris, J. Chem. Phys. 82, 541 (1985)], we believe that the evidence in such recent experiments is not strong enough to invalidate the CPS theory.

${ }^{15}$ R. Rossetti and L. E. Brus, J. Chem. Phys. 73, 572 (1980); 76, 1146 (1982).

${ }^{16}$ J. Arias, P. K. Aravind, and H. Metiu, Chem. Phys. Lett. 85, 404 (1982).

${ }^{17}$ P. T. Leung, Z. C. Wu, D. A. Jelski, and T. F. George, Phys. Rev. B 36, 1475 (1987).

${ }^{18}$ T. Rahman and A. A. Maradudin, Phys. Rev. B 21, 504 (1980).

${ }^{19}$ L. E. Brus, J. Chem. Phys. 73, 940 (1980); 74, 737 (1981).

${ }^{20}$ A. Campion, A. R. Gallo, C. B. Harris, H. J. Robota, and P. M. Whitmore, Chem. Phys. Lett. 73, 447 (1980).

${ }^{21}$ G. M. Goncher, C. A. Parsons, and C. B. Harris, J. Phys. Chem. 88, 4200 (1984).

${ }^{22}$ P. M. Whitmore, H. J. Robota, and C. B. Harris, J. Chem. Phys. 76, 740 (1982).

${ }^{23}$ P. T. Leung, T. F. George, and Y. C. Lee, J. Chem. Phys. 86, 7227 (1987).

${ }^{24}$ A. Maradudin and D. L. Mills, Phys. Rev. B 11, 1392 (1975).

${ }^{25}$ G. S. Agarwal, Phys. Rev. B 14, 846 (1976).

${ }^{26}$ Note that even in this CPS theory, some inconsistency exists since the damped nature of the dipole source is neglected when the $G$ functions are calculated, for a monochromatic incident field has been assumed as in the original Sommerfeld theory. This is justified since the molecular level widths $(\gamma)$ are in general much smaller than the molecular emission frequencies $(\omega)$. Nevertheless, this defect can be remedied by regarding the $\omega$ 's and $k$ 's as complex quantities in the original form of the theory. An assessment of such changes to the final results for the decay rate will be published in a future paper.

${ }^{27}$ Lord Rayleigh, Philos. Mag. 14, 70 (1907); U. Fano, J. Opt. Soc. Am. 31, 213 (1941).

${ }^{28}$ A. Marvin, F. Toigo, and V. Celli, Phys. Rev. B 11, 2777 (1975).

${ }^{29}$ F. Toigo, A. Marvin, V. Celli, and N. R. Hill, Phys. Rev. B 15, 5618 (1977).

${ }^{30}$ S. S. Jha, J. R. Kirtley, and J. C. Tsang, Phys. Rev. B 22, 3973 (1980).

${ }^{31}$ G. C. Brown, V. Celli, and M. Haller, Surf. Sci. 136, 381 (1984).

${ }^{32}$ B. Laks, D. L. Mills, and A. A. Maradudin, Phys. Rev. B 23, 4965 (1981); G. S. Agarwal, Opt. Commun. 14, 161 (1975).

${ }^{33} \mathrm{~A}$ similar adaptation of the MM theory to the calculation of the scattering intensities of light from admolecules at grating surfaces has been carried out by P. K. Aravind, E. Hood, and H. Metiu, Surf. Sci. 109, 95 (1981).

${ }^{34}$ Note that this is the most serious approximation made in the MM theory, as discussed further in Sec. V.

${ }^{35}$ All integrals involving $\delta\left(z^{\prime}\right)$ are understood to be evaluated according to the prescription of MM (Ref. 24) with Agarwal's modifications (Ref. 25).

${ }^{36}$ The approximation $\mathbf{k}^{(0)} \cong 0$ is $\operatorname{good}$ as long as $Q d / 2 \pi \ll<$, which is of course true in all our calculations that follow.

${ }^{37}$ The values for the dielectric constants for different metals are taken from the following references: P. B. Johnson and R. W. Christy, Phys. Rev. B 6, 4370 (1972), and Handbook of Optical Constants of Solids, edited by E. D. Palik (Academic, New York, 1985), p. 313.

${ }^{38}$ J. I. Gersten and A. Nitzan, J. Chem. Phys. 86, 3557 (1987); J. Opt. Soc. Am. B 4, 293 (1987).

${ }^{39}$ B. N. J. Persson and N. D. Lang, Phys. Rev. B 26, 5409 (1982); B. N. J. Persson and S. Anderson, ibid. 29, 4382 (1984); P. Avouris and B. N. J. Persson, J. Phys. Chem. 88, 837 (1984). 